\title{
Migraine with aura is associated with impaired colour vision: Results from the cross-sectional German DMKG headache study
}

2015, Vol. 35(6) 508-5I5

(C) International Headache Society 2014 Reprints and permissions: sagepub.co.uk/journalsPermissions.nav DOI: 10.1 I77/03331024|4547|36 cep.sagepub.com

(SAGE

\author{
TP Jürgens', K Berger ${ }^{2}$, A Straube ${ }^{3}$ and L Khil ${ }^{2}$
}

\begin{abstract}
Background: Hypersensitivity to light, noise and odour are pivotal clinical characteristics of migraine associated with enhanced cortical excitability and dysfunctional habituation. However, little is known about the integrity of basic sensory functioning in migraine on a population-based level.

Methods: A total of 129 participants with migraine ( 105 without aura, MwoA, 24 with aura, MA) and 522 healthy controls without headache 12 months prior to baseline were included from a sample of the DMKG study and underwent standardised clinical sensory testing of smell, taste, hearing and vision.

Results: After adjustment for age, sex, smoking status and history of head injuries, the chance of impaired colour perception was significantly higher in MA compared to controls (odds ratio, $O R=3.20 ; 95 \% \mathrm{Cl}=1.20-8.53$ ) and MwoA (OR $=3.62 ; 95 \% \mathrm{Cl}=1.3 \mathrm{I}-9.97)$. Compared to MwoA, MA also had an increased chance of smell $(\mathrm{OR}=3.20 ; 95 \% \mathrm{Cl}=0.98-10.42)$ and taste $(\mathrm{OR}=2.58 ; 95 \% \mathrm{Cl}=0.90-7.40)$ impairment.

Conclusions: In this cross-sectional, population-based study on sensory functioning in migraine participants, colour vision was impaired interictally in MA compared to MwoA and controls.
\end{abstract}

\section{Keywords}

Migraine, aura, sensory threshold, vision, headache

Date received: 2 April 20I4; revised: 19 May 20I4; II July 20I4; accepted: I3 July 2014

\section{Introduction}

Migraine is a complex disorder of sensory processing affecting different parts of the central nervous system (1). Photo- and phonophobia are landmarks for the diagnosis of migraine with (MA) and without aura (MwoA) (2). More recently, osmophobia has been recognised as a third relevant correlate of ictal - but also interictal - sensory dysregulation $(3,4)$. In recent studies the prevalence of photophobia was as high as $76.4 \%$, phonophobia $85.1 \%$ and osmophobia $47.7 \%$ among the participants (5).

However, only a limited number of studies have assessed the integrity of the involved sensory systems using qualitative or quantitative testing. These studies were mostly conducted at specialised headache centres, inferring the risk of a selection bias (6-11). While some epidemiological studies assessed both hypersensitivity to light, noise and smell as well as subjective sensory impairment (12), only very few have used objective testing such as in a door-to-door approach as in our study (13).

Therefore, participants in a large population-based study on primary headaches in Germany were analysed to elucidate whether thresholds of the visual, olfactory, gustatory and auditory system were altered in MA and MwoA patients.

\footnotetext{
'Department of Systems Neuroscience, University Medical Centre Hamburg-Eppendorf, Germany

${ }^{2}$ Institute of Epidemiology and Social Medicine, Westfälische Wilhelms-University Münster, Germany

${ }^{3}$ Department of Neurology, Ludwig-Maximilians University, Germany
}

\section{Corresponding author:}

Tim P Jürgens, Headache Clinic, Department of Systems Neuroscience, University Medical Center Hamburg-Eppendorf, Martinistrasse 52, D-20246 Hamburg, Germany.

Email: t.juergens@uke.de 


\section{Methods}

\section{Study procedure and population sample}

The Dortmund Health Study (DHS) is part of a large epidemiologic project funded by the German Migraine and Headache Society (DMKG). The DHS aimed to assess the prevalence of headache types, cardiovascular and other chronic diseases, and their impact on everyday life among those affected (14). The study was conducted 2003-2004 and included face-to-face interviews followed by standardised tests of sensory thresholds and functioning including smell, taste, hearing and vision. The detailed study procedure is described elsewhere (14). In short, a random sample of the total population stratified by age and sex was drawn from the population register of the city of Dortmund. Participation was restricted to ages 25 to 75 years.

The study was approved by the local ethics committee of the Medical Faculty of the University of Münster and the Westphalian Chamber of Physicians. Informed consent was obtained by all participants prior to inclusion into the study.

A total of 1312 participants took part in the interview-assessment and subsequent testing of sensory performance. For the present analysis migraine cases (participants with definite or probable migraine) and controls (participants without headaches during the 12-month period prior to the interview) were identified within the DHS $(n=797)$. Out of these we excluded participants with insufficient command of the German language $(n=122)$ and missing values in sensory performance testing $(n=24)$ summing up to a total of 651 participants.

\section{Headache assessment and classification}

A structured interview on the diagnosis of primary headaches was designed based on the International Headache Society's criteria for headache classification which were current at the time the study was planned and conducted (ICDH-2) (2). This interview allowed the assessment of the six- and 12- month period prevalence of migraine and tension-type headache $(14,15)$.

Participants who reported a headache during 12 months prior to the interview were subject to further questions about their headache symptoms (such as headache frequency, duration, character, location and presence of accompanying features and aura symptoms). Based upon these characteristics, the headache was classified as definite MwoA (IHS-code 1.1) or MA (IHS-code 1.2.1). If not all diagnostic criteria were met, the headache was classified as probable migraine (IHScode 1.6) without or with aura. Thus, the definition of migraine included probable $(n=48)$ or definite migraine $(n=81)$ diagnoses. In the present study,
$98 \%(n=47)$ with probable migraine did not fulfil IHS criterion B: "Headache attacks lasting 4-72 hours" as already described in Pfaffenrath et al. (14).

\section{Sensory testing}

Sensory functioning was assessed in four modalities: vision, smell, hearing and taste. An impairment of the respective modality was assessed and classified as follows:

Olfactory testing. Smell was tested using Sniffin' Sticks (12-stick version, Burghart, Wedel; Germany), which are pen-like odour-dispensing devices commonly used in clinical practice and research (16). The sticks enable tests of odour discrimination and odour identification (17). Participants had to identify 12 common odours in a multiple-forced choice test using a list of four descriptions for each odour $(18,19)$. Odours were presented to the participants by placing each of the 12 sticks subsequently in front of both nostrils for three seconds. Smell impairment (hyposmia or anosmia) was assumed if participants identified six or less out of the 12 odours (19).

Gustatory testing. Taste was tested in an identification task using the four basic tastes (sweet, sour, salty and bitter) at suprathreshold concentrations (sweet: sucrose, $1.5 \mathrm{~g}$; sour: citric acid, $0.75 \mathrm{~g}$; salty: sodium chloride, $1.125 \mathrm{~g}$; bitter: quinine hydrochloride, $7.5 \mu \mathrm{g}$; all test substances were dissolved in $15 \mathrm{~g}$ water) (19). Approximately $0.1 \mathrm{ml}$ of each tastant was sprayed on the middle of the participants' tongue. They were then asked to identify the taste according to a list with the four taste descriptors. After each taste, participants flushed their mouth with water (19). Taste impairment was assumed when participants did not identify all four tastes correctly. A sum score was created by adding 1 for each correctly identified gustatory sample (out of a maximum of 4). In addition, the self-rated taste intensity was measured on a scale from 1 (weak) to 10 (intensive). Participants were asked to rate the perceived intensity for each of the four tastes, resulting in a score from 1 to 40 . Then the average taste intensity was calculated as the mean intensity of all four tastes.

Visual testing. Monocular visual acuity was measured using a standard Snellen chart. If applicable, patients were tested wearing their current correction. Vision impairment was defined to be present if participants had a visual acuity of $80 \%$ or less. Colour perception was tested by the use of Ishihara plates (20). These charts present different numbers consisting of colour spots grounded on a different-coloured background, so that colour-deficient people cannot or just barely 
perceive the numbers (20). Participants were asked to identify the number presented on the colour chart. In total, 14 charts were presented out of which the average of correctly identified colour charts was estimated. If participants identified less than 13 colour charts, a colour-perception impairment was assumed.

Auditory assessment. Hearing was tested using a screening test with hearing thresholds of $30 \mathrm{~dB}$ by means of a portable device. Three frequencies $(1000 \mathrm{~Hz}, 2000 \mathrm{~Hz}$ and $4000 \mathrm{~Hz}$ ) were tested subsequently, starting with the right ear. A pure-tone of $30 \mathrm{~dB}$ was presented to the participant in a two-alternative forced-choice task. If the participant did not perceive the auditory stimulus, sound-pressure was increased to $40 \mathrm{~dB}$. This procedure was repeated up to a sound-pressure of $80 \mathrm{~dB}$. Then hearing impairment was assessed separately for both ears first. If the auditory threshold was greater than $30 \mathrm{~dB}$ in one of the three frequencies, auditory performance was considered moderately impaired. A severe impairment was assumed if the threshold was greater than $30 \mathrm{~dB}$ in at least two frequencies. The final definition of hearing impairment encompassed a combination of the auditory performance of both ears. If a moderate impairment was present in both ears, or if a severe impairment was present in at least one ear, the auditory function was defined as being impaired.

Combined sensory assessment. Since it was illustrated in other studies that sensory impairments overlap frequently $(19,21,22)$, we estimated the frequency of a multiple sensory impairment. We defined a multiple sensory impairment to be present if three out of the four investigated sensory impairments overlapped.

\section{Statistical analysis}

Continuous variables were described with means and standard deviations, and differences among groups were examined with analysis of variance (ANOVA).
Because taste and smell scores were not normally distributed, Kruskal-Wallis test was used instead. Differences in categorical variables were compared using chi-square test or Fisher's exact test (if cell number was 5 or less).

All impairment variables were dichotomous. Whether the chance of sensory impairment was higher in migraine compared to controls was tested by logistic regression. We calculated unadjusted as well as adjusted odds ratios (OR). The latter were adjusted for age, sex, smoking status and history of head injury since these variables were associated with either group, outcome or both. Adjusted group means were estimated by least square means using $t$-tests to identify differences between groups. All analyses were conducted using the statistical software SAS version 9.2.

\section{Results}

\section{Participant characteristics}

A total of 651 participants (522 controls and 129 cases) were analysed. As illustrated in Table 1, 105 out of the 129 migraineurs had MwoA and 24 MA. Participants with migraine were significantly younger than controls $(p<0.01)$. There were also more women in the migraine groups compared to controls $(p<0.01)$. The prevalence of current smokers $(p=0.84)$, diabetes $(p=0.11)$ or obesity $(p=0.30)$, defined as body mass index $(\mathrm{BMI}) \geq 30$ ), was not different between groups. However, there were more participants with depressive symptoms $(p<0.01)$ or a history of head injuries $(p<0.01)$ in the group of participants with migraine.

\section{Sensory assessment}

Impairment in at least one sensory modality was frequent but multiple sensory impairments were uncommon, as illustrated in Table 2. There was a group association for any or multiple impairments

Table I. Epidemiological characteristics.

\begin{tabular}{|c|c|c|c|}
\hline & Controls $(n=522)$ & MwoA $(n=105)$ & MA $(n=24)$ \\
\hline Age, mean (SD) & $58.2(12.1)$ & $46.7(11.4)$ & $45.5(11.5)$ \\
\hline Women,\% (n) & $43.0(227)$ & $82.2(88)$ & $79.2(19)$ \\
\hline Current smoker, \% (n) & $19.9(105)$ & $23.4(25)$ & $16.7(4)$ \\
\hline History of head injuries, \% ( $n$ ) & $5.9(31)$ & $12.2(13)$ & $20.9(5)$ \\
\hline BMI $\geq 30, \%(n)$ & $28.4(148)$ & $21.0(22)$ & $29.2(7)$ \\
\hline Diabetes, \% (n) & $9.8(5 \mathrm{I})$ & $3.8(4)$ & $4.2(\mathrm{I})$ \\
\hline Depressive symptoms ${ }^{\mathrm{a}}, \%(n)$ & $10.0(52)$ & $30.5(32)$ & $33.3(8)$ \\
\hline
\end{tabular}

MwoA: migraine without aura; MA: migraine with aura; SD: standard deviation; BMI: body mass index.

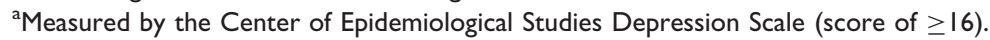


Table 2. Prevalence of sensory impairments.

\begin{tabular}{|c|c|c|c|}
\hline & $\begin{array}{l}\text { Controls } \\
(n=522)\end{array}$ & $\begin{array}{l}\text { MwoA } \\
(n=105)\end{array}$ & $\begin{array}{l}\text { MA } \\
(n=24)\end{array}$ \\
\hline Any sensory impairment, \% (n) & $88.3(46 I)$ & $75.2(79)$ & $95.8(23)$ \\
\hline Multiple sensory impairments, \% (n) & $29.3(153)$ & $7.6(8)$ & $29.2(7)$ \\
\hline Olfactory impairment, \% (n) & $24.3(127)$ & $10.5(\mathrm{II})$ & $25.0(6)$ \\
\hline Gustatory impairment, \% (n) & $20.8(109)$ & $14.3(15)$ & $29.2(7)$ \\
\hline Visual impairment, \% (n) & $35.1(183)$ & $28.5(30)$ & $20.8(5)$ \\
\hline Colour perception impairment, \% (n) & $56.5(295)$ & $47.6(50)$ & $75.0(18)$ \\
\hline Auditory impairment, \% (n) & $51.7(270)$ & $20.0(21)$ & $29.2(7)$ \\
\hline Self-perceived taste intensity, mean (SD) & $19.9(6.8)$ & $20.4(7.2)$ & $20.2(6.2)$ \\
\hline Smell score, mean (SD) & $10.2(1.8)$ & $10.9(1.1)$ & $10.3(2.2)$ \\
\hline (median (range)) & $(I I .0(12.0))$ & $($ II.0 (5.0)) & $(\mathrm{I} I .0(10.0))$ \\
\hline Taste score, mean (SD) & $3.7(0.7)$ & $3.8(0.5)$ & $3.7(0.6)$ \\
\hline (median (range)) & $(4.0(3.0))$ & $(4.0(3.0))$ & $(4.0(2.0))$ \\
\hline
\end{tabular}

MwoA: migraine without aura; MA: migraine with aura; SD: standard deviation.

$(p<0.01)$. In more detail, a significant association between group and olfactory $(p<0.01)$ as well as auditory $(p<0.01)$ and colour perception impairment $(p=0.03)$ but not visual $(p=0.31)$ or gustatory $(p=0.47)$ impairment could be found with a higher prevalence as shown in Table 2. The average self-perceived taste intensity did not differ between groups $(p>0.76)$. The same was true for the average taste score $(p=0.18)$. However, the smell score was significantly different between groups $(p<0.01)$.

After adjustment for age, sex, history of head injuries and smoking status, MA also had a higher and statistically significant chance of impaired colour vision compared to controls $(\mathrm{OR}=3.20 ; 95 \%$ confidence interval $(\mathrm{CI})=1.20-8.53)$ and MwoA $(\mathrm{OR}=3.62 ; 95 \% \mathrm{CI}=1.31-9.97)$ (see Table 3 for further details). Furthermore, MA showed a tendency to olfactory $(\mathrm{OR}=3.20 ; 95 \% \mathrm{CI}=0.98-10.42)$ and gustatory $(\mathrm{OR}=2.58 ; 95 \% \quad \mathrm{CI}=0.90-7.40) \quad$ impairment compared to MwoA, in line with high OR and large CI. The number of correctly identified tastants (sum score) and their subjective intensity did not differ between groups after adjustment.

\section{Association with photo-, osmo- and phonophobia}

In participants with migraine $79.8 \%(81.9 \%$ in MwoA and $70.8 \%$ in $\mathrm{MA}$ ) reported photophobia during attacks. Phonophobia was reported by $83.0 \%(83.8 \%$ and 79.2), while osmophobia was present in $24.8 \%$ $(24.8 \%$ in MwoA and $25.0 \%$ in MA). No relevant association was found between the presence of osmophobia and impaired olfactory perception $(\mathrm{OR}=0.98 ; 95 \% \mathrm{CI}=0.29-3.36)$, phonophobia and auditory impairment $(\mathrm{OR}=2.54 ; 95 \% \quad \mathrm{CI}=0.54$ 10.29) and photophobia and impaired colour vision $(\mathrm{OR}=1.43 ; 95 \% \mathrm{CI}=0.60-3.41)$.

\section{Discussion}

In this large population-based study a detailed assessment of headache type and sensory functions was conducted and the association of MA as well as MwoA and sensory functioning examined. After adjustment for potential confounders the chance of colour perception impairment was clearly higher in MA participants. Furthermore, we observed an increased chance of olfactory and gustatory impairment in MA participants even though this missed statistical significance. The prevalence of visual and auditory impairment, as well as the perceived intensity of basic tastes, did not differ among groups. There was also no significant association between olfactory function and the reported proportion of osmophobia, between auditory impairment and phonophobia and between colour vision and photophobia. Thus, our results do not support the idea of a general sensory dysfunction in migraine.

Osmophobia, which is associated with altered activity in the antero-superior temporal gyrus and the piriform cortex (23), was reported by $25 \%$ in our population, which corroborates findings of Kelman $(24.7 \%)$ in a sample of 673 migraine patients treated at a specialised headache centre (4). The only study using a face-to-face approach similar to ours detected osmophobia in $48 \%$ of patients (5). Prevalence found in other tertiary headache centres was higher: $46 \%$ by Blau and Solomon (3), 44\% by Zanchin and colleagues 
Table 3. Comparisons of sensory performance between groups.

\begin{tabular}{|c|c|c|c|}
\hline & & Raw OR (Cl) & Adjusted OR $(\mathrm{Cl})^{\mathrm{a}}$ \\
\hline \multirow[t]{3}{*}{ Olfactory impairment } & MwoA vs. control & $0.36(0.19-0.70)$ & $0.74(0.36-1.53)$ \\
\hline & MA vs. control & $1.03(0.40-2.67)$ & $2.38(0.83-6.83)$ \\
\hline & MA vs. MwoA & $2.85(0.93-8.69)$ & $3.20(0.98-10.42)^{\mathrm{b}}$ \\
\hline \multirow[t]{3}{*}{ Gustatory impairment } & MwoA vs. control & $0.63(0.35-1.14)$ & $0.81(0.43-1.55)$ \\
\hline & MA vs. control & $1.56(0.63-3.86)$ & $2.10(0.80-5.47)$ \\
\hline & MA vs. MwoA & $2.47(0.88-6.96)$ & $2.58(0.90-7.40)^{\mathrm{b}}$ \\
\hline \multirow[t]{3}{*}{ Auditory impairment } & MwoA vs. control & $0.23(0.14-0.39)$ & $0.70(0.38-1.30)$ \\
\hline & MA vs. control & $0.38(0.16-0.94)$ & I.34 (0.45-4.00) \\
\hline & MA vs. MwoA & $1.65(0.61-4.49)$ & $1.91(0.59-6.20)$ \\
\hline \multirow[t]{3}{*}{ Vision right eye } & MwoA vs. control & $0.52(0.29-0.91)$ & $0.72(0.38-I .35)$ \\
\hline & MA vs. control & $0.4 I(0.12-1.39)$ & $0.63(0.17-2.25)$ \\
\hline & MA vs. MwoA & $0.80(0.21-2.98)$ & $0.87(0.23-3.38)$ \\
\hline \multirow[t]{3}{*}{ Vision left eye } & MwoA vs. control & $0.93(0.56-1.53)$ & I.52 (0.85-2.7I) \\
\hline & MA vs. control & $0.44(0.13-1.5 I)$ & $0.80(0.22-2.92)$ \\
\hline & MA vs. MwoA & $0.48(0.13-1.74)$ & $0.53(0.14-2.02)$ \\
\hline \multirow[t]{3}{*}{ Colour perception } & MwoA vs. control & $0.70(0.46-1.10)$ & $0.89(0.60-1.4 I)$ \\
\hline & MA vs. control & $2.31(0.90-5.91)$ & $3.20(1.20-8.53)^{c}$ \\
\hline & MA vs. MwoA & $3.30(1.21-8.97)$ & $3.62(1.31-9.97)^{c}$ \\
\hline \multirow{5}{*}{$\begin{array}{l}\text { Self-perceived taste } \\
\text { intensity } \\
\text { Adjusted means } \\
\quad(95 \% \mathrm{Cl})\end{array}$} & MwoA vs. control & $\begin{array}{l}20.4(19.1-2 \mid .7) \text { vs. } \\
19.9(19.3-20.5)\end{array}$ & $\begin{array}{l}19.8(|8.1-2| .4) \text { vs. } \\
20.2(\mid 9.0-21.3) ;\end{array}$ \\
\hline & & $p=0.46$ & $p=0.60$ \\
\hline & MA vs. control & $20.2(19.1-21.7)$ vs. & 19.7 (I6.8-22.5) vs. \\
\hline & & $\begin{array}{l}19.9(19.3-20.5) \\
p=0.82\end{array}$ & $\begin{array}{l}20.2(19.0-21.3) \\
p=0.74\end{array}$ \\
\hline & MA vs. MwoA & $\begin{array}{l}20.2(17.5-23.0) \text { vs. } \\
20.4(19.1-1.7) \\
p=0.89\end{array}$ & $\begin{array}{l}19.7(\mid 6.8-22.5) \text { vs. } \\
19.8(|8 .|-2 \mid .4) ; \\
p=0.96\end{array}$ \\
\hline
\end{tabular}

$\mathrm{Cl}$ : confidence interval; MwoA: migraine without aura; MA: migraine with aura; OR: odds ratio; $\mathrm{Cl}$ : confidence interval.

${ }^{a}$ Adjusted for age, sex, smoking status and history of head injuries.

${ }^{\mathrm{b}}$ Relevant association due to high OR and large $\mathrm{Cl}$ despite missing statistical significance by a narrow margin.

'Significant results (with $p<0.05$ ).

(24) and $62 \%$ in Taiwan (25). Highest rates were found in $71 \%$ in a Japanese cohort of MA patients and in $49 \%$ of MwoA (26).

Little is known on the integrity of the olfactory system in migraine. Snyder and Drummond found an impaired interictal olfaction in MA and MwoA patients compared to healthy controls using vanillin (6). Similarly, Hirsch reported hypo- or anosmia in $18 \%$ of 76 patients with migraine (27). As a limitation, a control group was not included and only pyridine odour threshold was tested with a putative trigeminal co-activation. While both studies corroborate our findings, it was specific only for MA patients in our study.

Only recently Marmura and colleagues reported impaired olfactory ability during migraine attacks in MwoA and MA patients as determined with the University of Pennsylvania Smell Identification Test (UPSIT) (28). While baseline olfaction did not differ from a healthy control group, UPSIT scores shortly after an attack were numerically lower than at baseline. In line with our observation, the majority of patients $(66 \%)$ with a relevant impairment of olfaction suffered MA.

In contrast, olfaction among 80 Japanese migraine patients in a neurological outpatient service did not differ from 30 controls, while aversive ratings for certain scents were significantly higher among migraine patients, especially those with MA (26). Interestingly, both phenomena were not associated in our cohort as well, which supports the concept of two different and independent pathophysiologic mechanisms.

As olfactory input underlies serotonergic modulation in the olfactory bulb via the $5 \mathrm{HT}_{2 \mathrm{c}}$ receptor (29), serotonergic dysregulation as part of migraine biology could explain hyposmia. Alternatively, altered mitochondrial $\mathrm{Ca} 2+$ signalling in the olfactory cortex 
which was identified as a crucial element in olfactory signalling (30) could be causal.

In a first study from a specialised headache centre, self-reported taste abnormalities were found in $24.6 \%$ of MA and MwoA patients (4). In a subsequent report of Kelman and Tanis, self-reported taste abnormalities were present in 827 of $1025(80.7 \%)$ MA and MwoA patients (7). However, it was not differentiated between taste impairment and gustatory hypersensitivity and no quantitative testing was performed. Approximately $90 \%$ of what is putative gustatory impairment can actually be contributed to olfactory impairment (31). This emphasizes the importance of sensory testing, especially as anosmic patients are often unaware of their situation (31).

Saisu and colleagues were the first to examine interictal differences in taste by means of standardised testing in migraine patients and controls and found no differences in the prevalence of hypo- or hypergeusia (26). In contrast, the higher ORs for impaired taste in MA in our study imply a reduced taste perception which may in part be explained by the different methodology. In the former clinic-based study, no adjustments for potential confounders were made and the classification of taste anomalies was different.

In a Belgian questionnaire-based study $(n=134)$ on interictal sensory symptoms in migraine, more patients indicated reduced visual acuity (14.2\%) and nocturnal vision $(14.9 \%)$ in the headache-free interval than an increased visual acuity $(9.7 \%)$ and improved colour vision $(9.0 \%)$ (12). Patients with at least one visual change were more likely to suffer from ictal photophobia. As a limitation, no objective sensory testing was conducted. These findings are in contrast to a large study from the 1970s which assessed uncorrected and (if needed) corrected visual acuity, the presence of latent and manifest squint for both near and distant vision and convergence as well as accommodation (13) in a total of 168 men and 246 women from a random population sample from Wales. Apart from hyperphoria in near vision no significant differences between participants with and without migraine could be identified. Likewise, a relevant interictal reduction of visual acuity was not found in another smaller sample (32). Our data thus add evidence that visual acuity is not altered in migraine patients.

However, more complex examinations of the visual system yielded subtle differences between migraine patients and controls. Harle and Evans found an impaired stereopsis and a minutely increased prevalence of heterophoria and aligning prism as compared to controls (8). In addition, they identified an increased risk of pupil anomalies, visual field defects and pattern glare (33). Diminished interictal sensitivity in short-wavelength automated perimetry has also been found in 50\% of MA and MwoA patients (32).

Anomalies in colour vision have been described by Shepherd, who reported deficient colour discrimination in migraine patients attributed to S-cone mediated detection of light at short wavelengths corresponding to blue light $(9,10)$. In contrast, in our sample Ishihara plates were used, not allowing further assessment of tritanopia (respectively blue vision). It is important to note that our results are supported by the male prevalence in the control group since impaired colour vision is more prevalent in males because of $\mathrm{x}$-chromosomal transmission. This could point to a disturbance in the parvocellular system as part of the primary visual cortex in areas rich with mitochondrial cytochrome oxidases where blobs and interblobs are located which play a crucial role in the complex process of colour vision (34). As a mitochondrial deficit of energy metabolism has been suspected in MA patients (35-37), it is tempting to assume an altered mitochondrial energy metabolism in the visual cortex of MA patients. Similar to our findings in the olfactory system, impairment of colour vision does not correlate with photophobia, suggesting two different pathophysiologic mechanisms.

Photophobia has been associated with retinal pathways not involved in image-formation which modulate the activity of dura-sensitive thalamocortical neurons (38). The prevalence of photophobia in our cohort is similar to that in a multicentre study from 12 Latin American urban communities using a face-to-face questionnaire (5).

Our findings of normal auditory thresholds in a representative population sample from Germany are supported by a controlled study on a sample of 58 patients with migraine (mainly MwoA) from a headache outpatient clinic from Egypt compared to 40 healthy controls (11). However, up to two-thirds of the migraine patients had at least one anomaly in the advanced electrophysiological testing including auditory brainstem response, transient evoked otoacoustic emissions and distortion product otoacoustic emissions. Likewise, Bolay and colleagues could not detect any significant difference in pure tone audiometry and speech discrimination scores between 53 migraine patients (37 with MwoA) and 41 healthy controls (39), similar to another study from Turkey (40). But, on a subclinical level a contralateral suppression of transiently evoked otoacoustic emissions was found.

In a long-term follow-up (median nine years) of a cohort of 61 patients with vestibular migraine from a specialised centre, a mild bilateral sensorineural hearing loss was present in $18 \%$ of the patients (41), which suggests a higher risk of auditory impairment in 
patients with a previous history of vestibulocochlear dysfunction.

As for phonophobia, the prevalence in our sample was similar to the findings of the community-based study from Latin America (both around 80\%). In these individuals, a brainstem dysfunction at the medial olivocochlear complex or a disturbed synaptic transmission between outer cochlear hair cells and olivocochlear efferents could be causal for the generation of phonophobia.

\section{Limitations}

As group sizes became relatively small for the MA group after exclusion of participants without sufficient knowledge of the German language, we cannot exclude that analyses were partly underpowered.

The sensory testing paradigm was chosen to allow mobile testing by specifically trained interviewers without a medical background as this was the only feasible setup for a large scale epidemiological study with a broad range of sensory modalities covered. As a limitation of this mostly binary classification into abnormal or normal sensory functioning, subtle subclinical anomalies could have been missed as shown by other studies.

Our controls tended to be older and suffer from diabetes more frequently, therefore, worse sensory performance than in the migraine group would be expected. As this is clearly not the case, it strongly supports the validity of our findings.

\section{Conclusion}

In our study, colour vision was significantly impaired in MA patients in a population-based sample. However, general basic sensory functioning was largely unimpaired in our representative sample without evidence for a general sensory dysfunction in migraine present between attacks.

\section{Clinical implications}

- In this population-based study, colour vision as well as smell and taste were altered in participants suffering from migraine with aura (MA) compared to those with migraine without aura (MwoA) and healthy controls.

- Visual acuity and basic performance of the auditory system did not differ between these groups; therefore, no evidence for a clinically relevant general interictal sensory dysfunction in migraine was found.

- Disturbances of colour vision in migraine have been reported before and may be due to a mitochondrial dysfunction in the primary visual cortex in MA patients.

\section{Funding}

This research was supported by grants of equal share from the German Migraine and Headache Society (DMKG) and a consortium formed by Almirall, Astra-Zeneca, BerlinChemie, Boehringer Ingelheim Pharma, Boots Healthcare, GlaxoSmithKline, Janssen Cilag, McNeil Pharmaceuticals, MSD Sharp \& Dohme and Pfizer.

\section{Conflicts of interest}

TPJ has received grants and honoraria from MSD, Pfizer, Allergan and Autonomic Technologies Inc.

AS has received honoraria from Allergan, Berlin Chemie, Desitin, MSD, Pfizer, and St Jude, and grants from the German Science Council, the German Secretary of Education and the Kröner-Fresenius Foundation.

Dr Berger has received research support for the conduction of the DMKG Headache Study; unrestricted grants of equal share from the German Migraine and Headache Society and a consortium formed by Almirall, Astra-Zeneca, BerlinChemie, Boehringer Ingelheim Pharma, Boots Healthcare, GlaxoSmithKline, Janssen Cilag, McNeil Pharmaceuticals, MSD Sharp \& Dohme, and Pfizer to the University of
Muenster; for the Course of Restless Legs Syndrome Study unrestricted grants from the German Restless Legs Society and a consortium formed by Boehringer Ingelheim Pharma, Mundipharma Research, Neurobiotec, UCB (Schwarz Pharma) and Roche Pharma to the University of Muenster; from the German Minister of Research and Education for several research projects within the German Competence Net Stroke and an ongoing cohort study on depression and subclinical arteriosclerosis.

LK has nothing to declare.

\section{References}

1. Sprenger T and Goadsby PJ. Migraine pathogenesis and state of pharmacological treatment options. BMC Med 2009; 7: 71 .

2. Headache Classification Committee of the International Headache Society. The International Classification of Headache Disorders, 2nd edition. Cephalalgia 2004; 24: 1-160.

3. Blau JN and Solomon F. Smell and other sensory disturbances in migraine. J Neurol 1985; 232: 275-276.

4. Kelman L. The place of osmophobia and taste abnormalities in migraine classification: A tertiary care study of 1237 patients. Cephalalgia 2004; 24: 940-946. 
5. Morillo LE, Alarcón F, Aranaga N, et al. Clinical characteristics and patterns of medication use of migraneurs in Latin America from 12 cities in 6 countries. Headache 2005; 45: 118-126.

6. Snyder RD and Drummond PD. Olfaction in migraine. Cephalalgia 1997; 17: 729-732.

7. Kelman L and Tanis D. The relationship between migraine pain and other associated symptoms. Cephalalgia 2006; 26: 548-553.

8. Harle DE and Evans BJW. The correlation between migraine headache and refractive errors. Optom Vis Sci 2006; 83: 82-87.

9. Shepherd AJ. Colour vision in migraine: Selective deficits for S-cone discriminations. Cephalalgia 2005; 25: 412-423.

10. Shepherd AJ. Color vision but not visual attention is altered in migraine. Headache 2006; 46: 611-621.

11. Hamed SA, Youssef AH and Elattar AM. Assessment of cochlear and auditory pathways in patients with migraine. Am J Otolaryngol 2012; 33: 385-394.

12. Amery WK, Waelkens $J$ and Vandenbergh V. The sensorium of the migraineur. Ital J Neurol Sci 1988; 9: 539-545.

13. Waters WE. Headache and the eye. A community study. Lancet 1970; 2: 1-4.

14. Pfaffenrath V, Fendrich K, Vennemann M, et al. Regional variations in the prevalence of migraine and tensiontype headache applying the new IHS criteria: The German DMKG Headache Study. Cephalalgia 2009; 29: $48-57$.

15. Winter AC, Hoffmann W, Meisinger C, et al. Association between lifestyle factors and headache. $J$ Headache Pain 2011; 12: 147-155.

16. Kobal G, Klimek L, Wolfensberger M, et al. Multicenter investigation of 1,036 subjects using a standardized method for the assessment of olfactory function combining tests of odor identification, odor discrimination, and olfactory thresholds. Eur Arch Otorhinolaryngol 2000; 257: 205-211.

17. Hummel T, Sekinger B, Wolf SR, et al. 'Sniffin' sticks': Olfactory performance assessed by the combined testing of odor identification, odor discrimination and olfactory threshold. Chem Senses 1997; 22: 39-52.

18. Hummel T, Konnerth CG, Rosenheim K, et al. Screening of olfactory function with a four-minute odor identification test: Reliability, normative data, and investigations in patients with olfactory loss. Ann Otol Rhinol Laryngol 2001; 110: 976-981.

19. Vennemann MM, Hummel T and Berger K. The association between smoking and smell and taste impairment in the general population. J Neurol 2008; 255: 1121-1126.

20. Swanson WH and Cohen JM. Color vision. Ophthalmol Clin North Am 2003; 16: 179-203.

21. Fischer ME, Cruickshanks KJ, Klein BEK, et al. Multiple sensory impairment and quality of life. Ophthalmic Epidemiol 2009; 16: 346-353.

22. Michikawa T, Nishiwaki Y and Takebayashi T. Are you conscious of any age-related taste impairment? Prevalence of and factors associated with taste impairment in Japan. J Am Geriatr Soc 2011; 59: 951-953.
23. Demarquay G, Royet JP, Giraud P, et al. Rating of olfactory judgements in migraine patients. Cephalalgia 2006; 26: 1123-1130.

24. Zanchin G, Dainese F, Trucco M, et al. Osmophobia in migraine and tension-type headache and its clinical features in patients with migraine. Cephalalgia 2007; 27: 1061-1068.

25. Wang Y-F, Fuh J-L, Chen S-P, et al. Clinical correlates and diagnostic utility of osmophobia in migraine. Cephalalgia 2012; 32: 1180-1188.

26. Saisu A, Tatsumoto M, Hoshiyama E, et al. Evaluation of olfaction in patients with migraine using an odour stick identification test. Cephalalgia 2011; 31: 1023-1028.

27. Hirsch AR. Olfaction in migraineurs. Headache 1992; 32: 233-236.

28. Marmura MJ, Monteith TS, Anjum W, et al. Olfactory function in migraine both during and between attacks. Cephalalgia. Epub ahead of print 19 March 2014. DOI: 0333102414527014.

29. Petzold GC, Hagiwara A and Murthy VN. Serotonergic modulation of odor input to the mammalian olfactory bulb. Nat Neurosci 2009; 12: 784-791.

30. Fluegge D, Moeller LM, Cichy A, et al. Mitochondrial $\mathrm{Ca}(2+)$ mobilization is a key element in olfactory signaling. Nat Neurosci 2012; 15: 754-762.

31. Hirsch AR. Osmophobia and taste abnormality in migraineurs: A tertiary care study. Headache 2005; 45: 763-764. (author reply 764).

32. McKendrick AM, Vingrys AJ, Badcock DR, et al. Visual field losses in subjects with migraine headaches. Invest Ophthalmol Vis Sci 2000; 41: 1239-1247.

33. Harle DE and Evans BJW. The optometric correlates of migraine. Ophthalmic Physiol Opt 2004; 24: 369-383.

34. Gegenfurtner KR and Kiper DC. Color vision. Annu Rev Neurosci 2003; 26: 181-206.

35. Barbiroli B, Montagna P, Cortelli P, et al. Complicated migraine studied by phosphorus magnetic resonance spectroscopy. Cephalalgia 1990; 10: 263-272.

36. Barbiroli B, Montagna $\mathrm{P}$, Cortelli $\mathrm{P}$, et al. Abnormal brain and muscle energy metabolism shown by $31 \mathrm{P}$ magnetic resonance spectroscopy in patients affected by migraine with aura. Neurology 1992; 42: 1209-1214.

37. Reyngoudt H, Achten E and Paemeleire K. Magnetic resonance spectroscopy in migraine: What have we learned so far? Cephalalgia 2012; 32: 845-859.

38. Noseda R, Kainz V, Jakubowski M, et al. A neural mechanism for exacerbation of headache by light. Nat Neurosci 2010; 13: 239-245.

39. Bolay H, Bayazit YA, Gündüz B, et al. Subclinical dysfunction of cochlea and cochlear efferents in migraine: An otoacoustic emission study. Cephalalgia 2008; 28: 309-317.

40. Bayazit Y, Yilmaz M, Mumbuç S, et al. Assessment of migraine-related cochleovestibular symptoms. Rev Laryngol Otol Rhinol (Bord) 2001; 122: 85-88.

41. Radtke A, von Brevern M, Neuhauser H, et al. Vestibular migraine: Long-term follow-up of clinical symptoms and vestibulo-cochlear findings. Neurology 2012; 79: 1607-1614. 